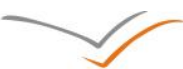

\title{
VERSITA
}

\section{A Simple Discrete Approximation for the Renewal Function}

\author{
Alenka Brezavšček \\ University of Maribor, Faculty of Organizational Sciences, Kranj, Slovenia
}

\begin{abstract}
Background: The renewal function is widely useful in the areas of reliability, maintenance and spare component inventory planning. Its calculation relies on the type of the probability density function of component failure times which can be, regarding the region of the component lifetime, modelled either by the exponential or by one of the peak-shaped density functions. For most peak-shaped distribution families the closed form of the renewal function is not available. Many approximate solutions can be found in the literature, but calculations are often tedious. Simple formulas are usually obtained for a limited range of functions only. Objectives: We propose a new approach for evaluation of the renewal function by the use of a simple discrete approximation method, applicable to any probability density function. Methods/Approach: The approximation is based on the well known renewal equation. Results: The usefulness is proved through some numerical results using the normal, lognormal, Weibull and gamma density functions. The accuracy is analysed using the normal density function. Conclusions: The approximation proposed enables simple and fairly accurate calculation of the renewal function irrespective of the type of the probability density function. It is especially applicable to the peak-shaped density functions when the analytical solution hardly ever exists.
\end{abstract}

Keywords: component, failure times, probability density function, random failures, wear-out failures, renewal function, approximation

JEL classification: C02, C44

Paper type: Research article

Received: 21, September, 2012

Revised: 29, November, 2012

Accepted: 24, December, 2012

Citation: Brezavšček, A. (2013). "A Simple Discrete Approximation for the Renewal Function", Business Systems Research, Vol. 4, No. 1, pp. 65-75.

DOI: 10.2478/bsri-2013-0006

\section{Introduction}

The renewal function $H(t)$ plays an important role in the areas of reliability, maintenance and spare parts inventory planning (see e.g. Sheikh and Younas, 1985; Barlow, Proschan, and Hunter, 1996; Gertsbakh, 2000; Brezavšček, 2011). The calculation of $H(t)$ relying on the type of the probability density function of inter-renewal times. In the reliability and maintainability applications this function is given by the probability density function of component failure times, usually denoted by the symbol $f(t)$. However, it is not possible to obtain $H(t)$ analytically for any type of the function $f(t)$. For most peak-shaped distribution families the closed form of $H(t)$ is not obtainable.

Many different approaches have been developed in the literature to approximate or numerically compute the renewal function. Chaudhry (1995), Garg and Kalagnanam (1998), Cui and Xie (2003), Hu (2006) or Politis and Koutras (2006) give a comprehensive review of the approaches for computing the renewal function. They found out that there are many 
methods to approximate the renewal function such as the extended cubic-splining algorithm, the generating function algorithm, and power series expansion (e.g. Robinson, 1997). The first method is not simple to implement, the second method is time consuming for large values of time, while the third method is distribution specific. Therefore, a simple and general approximation for calculating the renewal function is desired. The approximation should meet the requirements of simplicity (i.e. it could be used without a need of further numerical computation), accuracy (i.e. it should enable fairly accurate estimation of $H(t)$ for sufficiently large $t$ ), and applicability (i.e. it should be applicable for more distribution families rather than a specific distribution). Though some efforts have been made to develop such approximations (e.g. Smeitink and Dekker, 1990; Ayhan, Limon-Robles and Wortman, 1999; Bebbington, Davydov and Zitikis, 2007; Jiang, 2010) it appears that simple approximate formulas are obtained for a limited range of functions $f(t)$ only.

In this paper we develop a discrete approximation which enables a simple calculation of the renewal function irrespective of the type of the component failure time distribution function. The proposed approximation is especially useful for the peak-shaped density functions when the analytical solution of the renewal function hardly ever exists. The usefulness of the approximation is proved through some numerical results considering the normal, lognormal, Weibull and gamma density functions, while the accuracy of the approximation is analyzed using the normal density function.

\section{Theoretical framework}

\section{Probability density functions of component failure times}

Technical systems such as industrial systems consist of a number of components of different types. During the system operation its components fails. Time to failure of a component of a given type is a random variable distributed according to the probability density function $f(t)$.

The behaviour of the function $f(t)$ depends on the region of the component lifetime. From the reliability theory it is known that the lifetime of a component can be roughly divided into three regions: the region of early failures, the region of random failures (also called the region of the normal operation) and the region of wear-out failures. Early failures are usually detected and eliminated by screening or burn-in tests before the components are put into operation. The operating period of the component thus comprises only the region of random failures and the region of wear-out failures. Random failures are mainly due to inherent slowacting defects in the component, or to sudden excessive loading. The main failure mechanism in the wear-out region is deterioration of the component materials. The region of random failures is characterized by monotonically decreasing function $f(t)$, while in the region of wear-out failures the distribution of component failure times follows a peak-shaped curve. In Figure 1, the general form of the function $f(t)$ during the component lifetime is presented. To the side, the instantaneous failure rate $\lambda(t)$ is also shown. It can be seen that during the region of random failures $\lambda(t)$ has approximately constant value $\lambda$, simply called the failure rate. During the wear-out region, the instantaneous failure rate $\lambda(t)$ increases with time.

The renewal process and the renewal function

When the system during its operation fails, a failed component needs to be replaced by a new one as soon as possible. If the replacement time is negligible, the process of consecutive corrective replacements of a particular component can be modelled by an ordinary renewal process.

A realization of a renewal process consists of a series of point events (renewals) occurring singly in time and completely randomly. When all inter-renewal times (in our case times to component failure) are independent identically distributed random variables, the renewal process is called ordinary (e.g. Cox, 1970; Nakagawa, 2011). 
Figure 1

The Form of the Probability Density Function $f(t)$ of Component Failure Times and the Instantaneous Failure Rate $\lambda(t)$

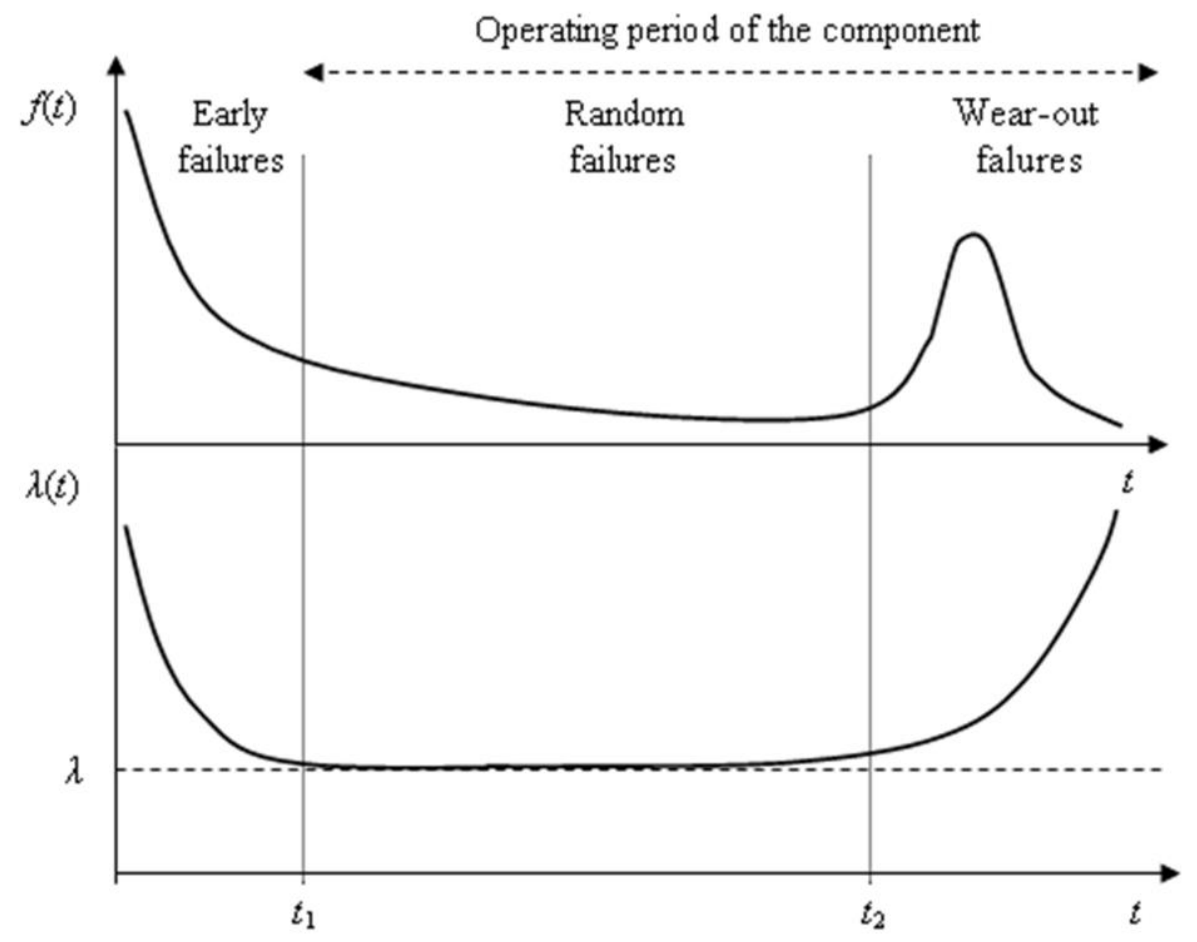

Source: Author's illustration

The essential characteristic of an ordinary renewal process is the renewal function $H(t)$, defined as the expected number of renewals of a single component in the interval $(0, t)$ (e.g. Cox, 1970; Beichelt, 2006; Nakagawa, 2011):

$H(t)=E[N(t)]$

where $N(t)$ represents the number of component renewals during the interval $(0, t)$.

The renewal function $H(t)$ can be obtained using to the equation (e.g. Cox, 1970; Beichelt, 2006; Nakagawa, 2011):

$H(t)=\sum_{r=1}^{\infty} F_{r}(t)$

with $F_{0}(t)=1$ and $F_{1}(t)=F(t)=\int_{0}^{t} f(x) d x$. The symbol $F_{r}(t)$ in (1) represents the $r$-fold convolution integral of the cumulative distribution function $F(t)$ :

$F_{r}(t)=\int_{0}^{t} F_{r-1}(t-x) d F(x), r \geq 2$

A simple solution of (1) is available for some specific types of $f(t)$ only. 
Theoretically, the calculation of the renewal function $H(t)$ according to (1) can be simplified using the Laplace transformations because there is a simple relation between the Laplace transform of a convolution and the Laplace transforms of the two functions being convoluted (e.g. Cox, 1970; Beichelt, 2006).

Taking into account the relations $L\{f(t)\}=f^{*}(s), \quad \mathrm{L}\{F(t)\}=\frac{f^{*}(s)}{s}$, $\mathrm{L}\left\{F_{r}(t)\right\}=\frac{\left[f^{*}(s)\right]^{r}}{s}$, the Laplace transform $H^{*}(s)$ of the renewal function is easily derived from (1) in the following form:

$H^{*}(s)=\frac{1}{s} \frac{f^{*}(s)}{1-f^{*}(s)}$

The renewal function $H(t)$ is then obtained by the inversion of the expression (2):

$H(t)=L^{-1} H^{*}(s)$

Unfortunately, when the function $f(t)$ belongs to one of the peak-shaped distribution families, $f^{*}(s)$ is not always available. Even if $f^{*}(s)$ is available the exact inversion of $H^{*}(s)$ in a simple form can hardly ever be obtained.

Another presentation of the renewal function is provided by the so called renewal equation (e.g. Tijms, 2003; Nakagawa, 2011):

$H(t)=F(t)+\int_{0}^{t} H(t-x) f(x) d x$

Calculation of the renewal function for different types of $f(t)$

It is clear that the calculation of $H(t)$ relies on the type of the probability density function $f(t)$ of component failure times. It follows from Figure 1 that an appropriate mathematical model for $f(t)$ in the region of random failures is the exponential density function, while in the region of wear-out failures the function $f(t)$ has got a peak-shaped form which is usually modelled by the normal, lognormal, Weibull or gamma density function (see e.g. O'Connor, 2011). We shall briefly discuss the properties of these types of density functions from the viewpoint of the renewal function calculation.

Exponential $f(t)$

The exponential density function is

$f(t)=\lambda e^{-\lambda t} \quad t>0, \lambda>0$

where the parameter $\lambda$ represents the constant failure rate. For this type of $f(t)$ the calculation of $H(t)$ is trivial. The analytical solution can be simply derived using the Laplace transform of $f(t)$ and equations (2) and (3). We obtain $H(t)=\lambda t$.

Normal $f(t)$

The normal density function is given by the expression 
$f(t)=\frac{1}{\sigma \sqrt{2 \pi}} e^{-\frac{1}{2}\left(\frac{t-\mu}{\sigma}\right)^{2}}-\infty<t<\infty,-\infty<\mu<\infty, \sigma>0$

with the parameters mean $\mu$ and standard deviation $\sigma$.

The normal density function, being defined in the interval $(-\infty, \infty)$, is an appropriate mathematical model for $f(t)$ only if $\mu / \sigma>>1$. In practice, we can assume that this condition is fulfilled when $\mu / \sigma \geq 3$. The negative tail of the normal curve is then at most 0.0013. If $\mu / \sigma<3$, a truncated normal distribution can be used (see e.g. Johnson et al., 1994; Kottegoda and Rosso, 1997).

In the case of normal $f(t)$ the exact inversion of (2) is not obtainable, and the analytical solution for $H(t)$ does not exist. However, as the $r$-fold convolution of the normal distribution function $F(t)$ with the parameters $\mu$ and $\sigma$ is also a normal distribution function with the parameters $r \mu$ and $\sigma \sqrt{r}$, the numerical calculation of $H(t)$ according to (1) is rather simple.

Lognormal $f(t)$

The lognormal density function is

$$
f(t)=\frac{1}{t \sigma \sqrt{2 \pi}} e^{-\frac{1}{2}\left(\frac{\ln t-\mu}{\sigma}\right)^{2}} \quad t>0, \mu>0, \sigma>0
$$

where $\mu$ and $\sigma$ are mean and standard deviation of $\ln t$.

When $f(t)$ is lognormal the exact inversion of (2) is not obtainable, so the analytical solution for $H(t)$ does not exist. Unfortunately, the numerical calculation is not trivial because the closed form of $F_{r}(t)$ is not directly obtainable. Some approximate formulas can be found in the literature but calculations are pretty complex (see e.g. Barouch and Kaufman, 1976; Romeo, Da Costa and Bardou, 2003; Lam and Le-Ngoc, 2006).

Weibull $f(t)$

The two-parameter ${ }^{1}$ Weibull density function is

$$
f(t)=\frac{\beta}{\eta}\left(\frac{t}{\eta}\right)^{\beta-1} e^{-\left(\frac{t}{\eta}\right)^{\beta}} t \geq 0, \beta>0, \eta>0
$$

where $\beta$ is the shape parameter, and $\eta$ is the scale parameter.

If the shape parameter $\beta$ is equal to 1 , the Weibull density function becomes exponential, and the calculation of the renewal function is trivial. When $\beta>1$, the Weibull density function follows a peak-shaped form. In such a case the closed form of $F_{r}(t)$ is not available. Consequently, the numerical calculation of $H(t)$ is quite tedious (see e.g. Jiang, 2008). An

\footnotetext{
${ }^{1}$ In reliability theory, the three parameter Weibull density function is also used. The third parameter $\gamma,-\infty<\gamma<\infty$, is the location parameter. When $\gamma=0$ the density function starts at time $t=0$.
} 
exhaustive overview of different methods for numerical calculation of the renewal function when underlying distribution is Weibull can be found in Rinne (2009).

Gamma $f(t)$

The two-parameter ${ }^{2}$ gamma density function is

$$
f(t)=\frac{1}{\eta \Gamma(\beta)}\left(\frac{t}{\eta}\right)^{\beta-1} e^{-\frac{t}{\eta}} \quad t \geq 0, \beta>0, \eta>0
$$

where $\beta$ represents the shape parameter, $\eta$ is the scale parameter ${ }^{3}$, and $\Gamma($.$) denotes the$ gamma function 4 .

If $\beta=1$, the gamma density function is equal to the exponential density function, and the calculation of $H(t)$ is trivial. Similar to the Weibull density function, the gamma density function follows a peak-shaped form when $\beta>1$. The analytical solution for $H(t)$ only exists for some integer values of $\beta$ (e.g., $\beta=2$ or $\beta=3$ ). The numerical calculation of $H(t)$ according to (1) is simple for any value of $\beta$. Namely, the $r$-fold convolution of the gamma distribution function with the parameters $\beta$ and $\eta$ is also a gamma distribution function with the parameters $r \beta$ and $\eta$.

We can conclude that the analytical solution for $H(t)$ is trivial when $f(t)$ is exponential (i.e. components operate in the region of random failures) while the closed form of $H(t)$ is hardly ever available when the underlying density function is a peak-shaped (i.e. the components operate in the region of wear-out failures). The derivation of an approximate solution of $H(t)$ for a peak-shaped $f(t)$ has attracted the attention of many authors, but simple formulas are obtained for a limited range of functions $f(t)$ only. A more general approximate solution for $H(t)$ would be most desirable.

\section{The approximate solution for the renewal function}

Our aim is to derive an approximate solution for evaluating the renewal function which should meet the following requirements:

- Mathematical operations involved are simple.

- Satisfactory accuracy is achieved even for sufficiently large value of time.

- The solution is useful for any type of the probability density function of component failure times.

- Especially it is applicable to the peak-shaped probability density functions which are useful to describe the component failure time distribution in the wear-out region.

\footnotetext{
2 In reliability theory, the three parameter gamma density function is also used. The third parameter $\gamma,-\infty<\gamma<\infty$, is the location parameter. When $\gamma=0$ the density function starts at time $t=0$.

${ }^{3}$ When $\beta$ is integer the gamma density function becomes the Erlang density function. When the shape parameter is $\beta / 2$ ( $\beta$ is any integer) and the scale parameter is equal to 2 the gamma density function becomes the Chisquare density function.

${ }_{4} \Gamma(x)=\int_{0}^{\infty} z^{x-1} e^{-z} d z$
} 
According to our knowledge, such a solution has not been provided in the literature till now.

As a basis for the derivation of our approximation the recursive integral equation (4) is used. The main idea of the approximation is a discretization of the continuous time interval $(0, T)$ by its division to a number of subintervals of length $h$, as shown in Figure 2 . The length $h$ of subintervals within $(0, T)$ should be short enough to ensure that the probability of occurring more than one component failure during $h$ is negligible.

Figure 2

Discretization of the Interval $(0, T)$

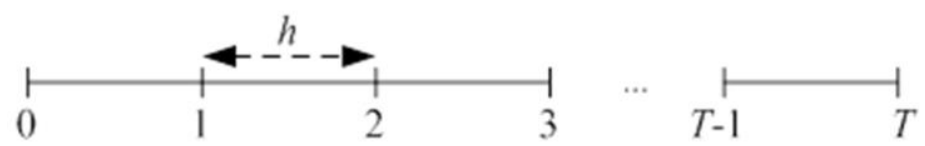

Source: Author's illustration

If the value $h$ is short enough, we can assume that component renewals during $(0, T)$ occur only in the time points $i, i=1,2, \ldots, T$ with the probability $p_{i}$. Considering this assumption our discrete approximation of (4) reads as follows:

$H(T)=\sum_{i=1}^{T} p_{i}+\sum_{i=1}^{T} H(T-i) p_{i} \quad T=1,2, \ldots$

$H(0)=0$

We define the probability $p_{i}$ in (5) by the integral

$p_{i}=\int_{i-1}^{i} f(t) d t, i=1,2, \ldots, \sum_{i=1}^{\infty} p_{i}=1$

This definition implies that the values of discrete and continuous cumulative distribution functions of inter-renewal times are equal since:

$\sum_{i=1}^{T} p_{i}=\sum_{i=1}^{T} \int_{i=1}^{i} f(t) d t=\int_{0}^{T} f(t) d t=F(T)$ (6)

Considering the equations (5) and (6) we get the following algorithm for estimating the value $H(T)$ of the renewal function in an arbitrary time $T$ :

$H(T)=F(T)+\sum_{i=1}^{T} H(T-i) \int_{i-1}^{i} f(t) d t \quad T=1,2, \ldots$ (7)

with the initial condition $H(0)=0$.

Since our approximation (7) involves the assumption that the renewals which can actually occur anywhere within the interval $(0, T)$ occur exactly at the time points $i, i=1,2, \ldots, T$, the value $H(T)$, calculated according to (7), is too low. The error introduced in such a way can 
be diminished by choosing sufficiently small value of $h$. When $h \rightarrow 0$ the value $H(T)$, calculated according to (7), converges to the exact value of the renewal function. However, decreasing of $h$ will improve the accuracy of our approximation (7) but also increase the number of recursive calculations. Therefore, the length $h$ of subintervals between two successive time points $i, i=1,2, \ldots, T$, is appropriate, when its shortening does not change the value of $H(T)$ over acceptable limits.

Because the mathematical operations in (7) are simple, the renewal function can be calculated easily for any value of time $T$ and regardless of the type of the function $f(t)$. Similar approximations, derived in a different way, have been proposed in Jardine (1973), Jardine and Tsang (2006) and van Noortwijk and van der Weide (2008).

\section{Numerical results}

To prove the usefulness of the approximation (7) some numerical results are given in Table 1. In the calculations, the normal, lognormal, Weibull and gamma density functions are considered.

Results from Table 1 prove that our approximation (7) is applicable to all types of density functions which are widely used in reliability studies to describe the function $f(t)$ in the wearout region. Although all of density functions considered are of peak-shaped form, the calculated values of $H(T)$ cannot be compared directly. In the case of the normal and the gamma density functions the values $H(T)$, calculated according (7), can be compared with the corresponding values, calculated according to (1). We can briefly conclude that our approximation gives fairly accurate results even for comparatively large values of time.

Table 1

Applicability of the Approximation (7) to Different Peak-Shaped Probability Density Functions, Widely Used in the Reliability Applications

\begin{tabular}{|c|c|c|c|c|c|c|c|}
\hline & $\bar{t}$ & $0.25 \mu$ & $0.5 \mu$ & $0.75 \mu$ & $\bar{\mu}$ & $2 \mu$ & $3 \mu$ \\
\hline \multirow{2}{*}{$\begin{array}{c}\text { Normal pdf } \\
\mu=45 \quad \sigma=15\end{array}$} & $\begin{array}{l}H(t) \text { calculated } \\
\text { according to (1) }\end{array}$ & 0.01233 & 0.06755 & 0.23068 & 0.51722 & 1.54166 & 2.55363 \\
\hline & $\begin{array}{l}H(t) \text { calculated } \\
\text { according to }(7)\end{array}$ & 0.01122 & 0.06674 & 0.22941 & 0.51392 & 1.53687 & 2.53418 \\
\hline \multirow{2}{*}{$\begin{array}{c}\text { Lognormal pdf } \\
\mu^{\prime}=5 \quad \sigma^{\prime}=0.3 \\
\Downarrow \\
\mu \approx 155 \quad \sigma \approx 48\end{array}$} & $\begin{array}{l}H(t) \text { calculated } \\
\text { according to (1) }\end{array}$ & - & - & - & - & - & - \\
\hline & $\begin{array}{l}H(t) \text { calculated } \\
\text { according to }(7)\end{array}$ & 4.20E-06 & 0.01600 & 0.20572 & 0.55834 & 1.54237 & 2.53594 \\
\hline \multirow{2}{*}{$\begin{array}{c}\text { Weiblull pdf } \\
\beta=3.5 \eta=200 \\
\Downarrow \\
\mu \approx 180 \sigma \approx 57\end{array}$} & $\begin{array}{l}H(t) \text { calculated } \\
\text { according to (1) }\end{array}$ & - & - & - & - & - & - \\
\hline & $\begin{array}{l}H(t) \text { calculated } \\
\text { according to }(7)\end{array}$ & 0.00539 & 0.05939 & 0.22488 & 0.51039 & 1.53147 & 2.53710 \\
\hline \multirow{2}{*}{$\begin{array}{c}\text { Gamma pdf } \\
\beta=2.9 \quad \eta=50 \\
\Downarrow \\
\mu=145 \quad \sigma \approx 85\end{array}$} & $\begin{array}{l}H(t) \text { calculated } \\
\text { according to }(1)\end{array}$ & 0.04395 & 0.20226 & 0.42552 & 0.67093 & 1.67240 & 2.67241 \\
\hline & $\begin{array}{l}H(t) \text { calculated } \\
\text { according to (7) }\end{array}$ & 0.04322 & 0.20129 & 0.42478 & 0.66968 & 1.66778 & 2.66436 \\
\hline
\end{tabular}

Source: Author's Calculations

The accuracy of the approximation (7) depends on the length $h$ of subintervals between two successive time points $i, i=1,2, \ldots, T$ (see Figure 2). To analyze the effect of shortening of $h$ to the accuracy of the calculated values we use the normal density function. In order to 
exclude the possible effect of the negative tail we present the results for the ratio $\mu / \sigma=4$. We compare the values of $H(T)$, calculated according to $(1)^{5}$, and the corresponding values, calculated according to (7). In calculations according to (7) different values of $h$ are considered. Results for the time span up to $3 \mu$ are shown in Figure 3.

\section{Figure 3}

The influence of the length $h$ of subintervals between two successive time points $i, i=1,2, \ldots, T$, on the accuracy of the approximation (7)

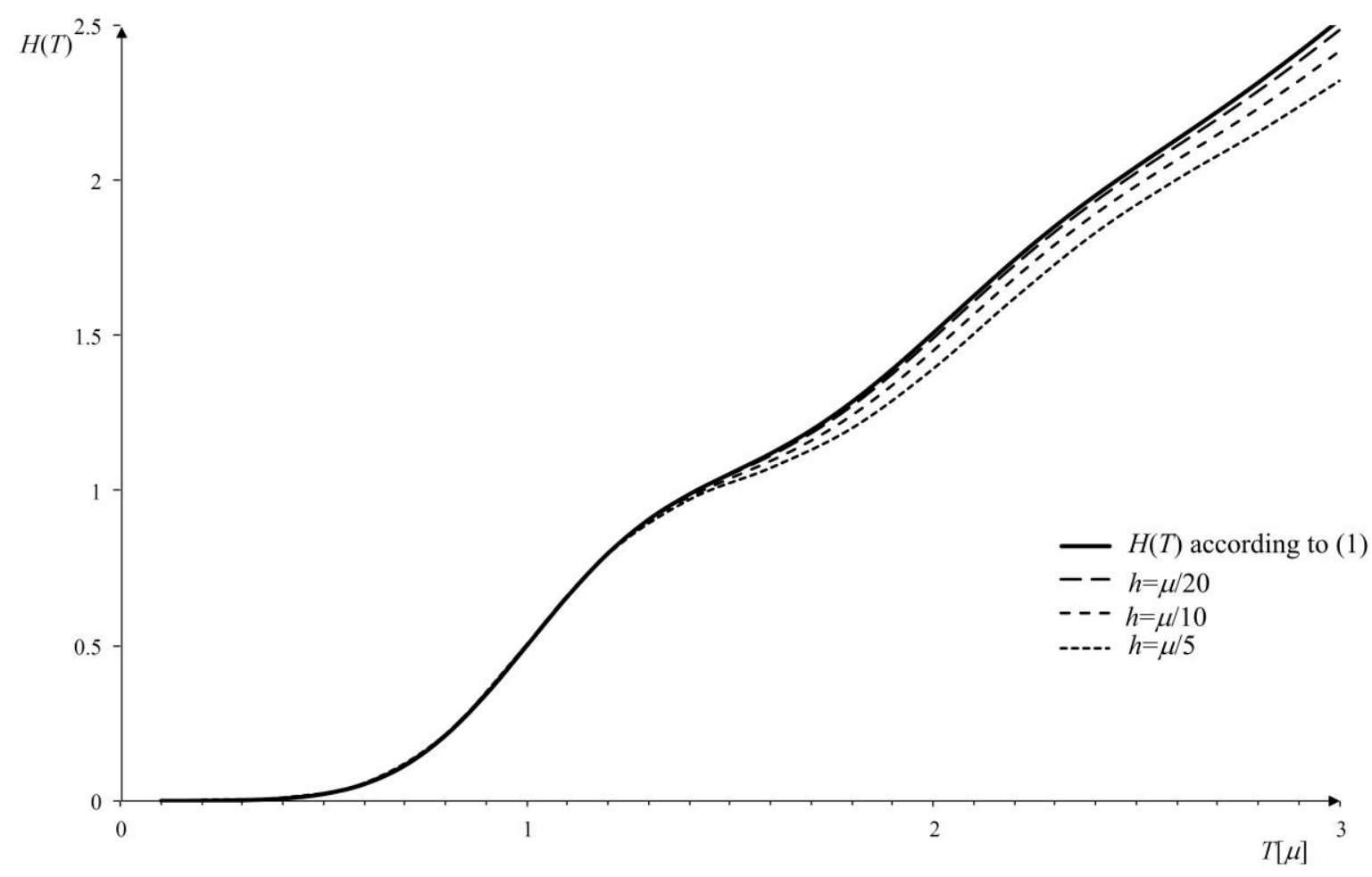

Source: Author's illustration

It can be seen from Figure 3 that for $T \leq 1.2 \mu$ all the curves overlap. This proves that for fairly short times our approximation (7) provides accurate results irrespective of the value of $h$ . When $T$ increases the error of $H(T)$ calculated using (7) increases. However, we can see that shortening of $h$ diminish the error efficiently. When $h=\mu / 30$ the error does not exceed $1 \%$ for times between $\mu$ and $2 \mu$. For smaller values of $h$ still better accuracy is obtained. For example, if $h=\mu / 50$ the error does not exceed $1 \%$ even for $T=3 \mu$.

\section{Conclusion}

The renewal function is an important characteristic, needed in the areas of reliability analyses, maintenance optimization and spare components inventory planning. Its calculation depends on the form of the probability density function of failure times during the region of component operation. In the region of random failures, the calculation of the renewal function is trivial because the probability density function of component failure times is

5The approximation $H(T) \approx \sum_{r=1}^{6} F_{r}(T)$ is used. The maximum error does not exceed 3E-6. 
described by the exponential density function. In the region of wear-out failures, the probability density function of component failure times exhibits a peak-shaped form. In that case, the calculation of the renewal function is in general difficult because the analytical solutions hardly ever exist. The derivation of an approximate solution of the renewal function for a peak-shaped failure time distribution has attracted the attention of many authors, but simple approximate formulas are obtained for a limited range of functions only.

In this paper, we have proposed a discrete approximation for estimating the value of the renewal function. The approximation is derived from the so called renewal equation. Due to simple mathematical operations involved, it enables a simple and fairly accurate calculation of the renewal function for any type of the probability density function of component failure times. It is especially applicable to the peak-shaped density functions when the analytical solutions hardly ever exist. To prove the usefulness of the approximation some numerical results are given considering the normal, lognormal, Weibull and gamma probability density functions. It is also shown that satisfactory accuracy of the approximation can be achieved by division of the time interval into sufficiently short subintervals.

Since the approximation proposed meets the requirements of the simplicity as well as the applicability to different types of peak-shaped density functions, the practical value of the approximation is significant. The approximation is useful everywhere in the reliability analysis and the maintenance policy optimization where the renewal function needs to be evaluated.

The main limitation of our approximation is the following: The accuracy of our approximation depends on the length $h$ of subintervals between two successive time points, where smaller $h$ ensures better accuracy (see Figure 2). However, the negative effect of the decreasing of $h$ results in the increasing of the number of recursive calculations. Consequently, when someone wants to calculate very accurate estimation of the renewal function in a very large value of time (i.e. $t>>\mu$ ), our approximation becomes time consuming, and therefore probably useless. However, it has been shown that within the acceptable limits of calculating time the error less than $1 \%$ in the time span up to $3 \mu$ can be achieved. In our opinion, for practical purposes this is good enough, even because a simple asymptotic formula for the renewal function (Cox, 1970; Beichelt, 2006) can be used for larger values of time.

\section{References}

1. Ayhan H., Limon-Robles, J., Wortman M. A. (1999), „An approach for computing tight numerical bounds on renewal functions", IEEE Transactions on Reliability, Vol. 48 No. 2, pp. 182-188.

2. Barlow, R. E., Proschan, F., Hunter, L. C. (1996). Mathematical Theory of Reliability, Philadelphia, SIAM.

3. Barouch, E., Kaufman, G. M. (1976), „On Sums of Lognormal Random Variables”. Working paper, Alfred P. Sloan School of Management, Cambridge, Massachusetts, available at http://dspace.mit.edu/bitstream/handle/1721.1/48703/onsumsoflognorma00baro.pdf / (10 June 2011).

4. Bebbington, M., Davydov, Y., Zitikis, R. (2007), "Estimating the renewal function when the second moment is infinite", Stochastic Models, Vol. 23 No.1, pp. 27 - 48.

5. Beichelt, F. (2006). Stochastic Processes in Science, Engineering And Finance, Boca Raton, Chapman \& Hall/CRC.

6. Brezavšček, A. (2011), ,Simple Stochastic Model for Planning the Inventory of Spare Components Subject to Wear-out", Organizacija, Vol. 44 No. 4, pp. $120-127$.

7. Chaudhry, M. L. (1995), "On computations of the mean and variance of the number of renewals: a unified approach", The Journal of the Operational Research Society, Vol. 46 No. 11, pp. 1352-1364.

8. Cox, D. R. (1970). Renewal Theory, London \& Colchester: Methuen. 
9. Cui, L., Xie, M. (2003), „Some normal approximations for renewal function of large Weibull shape parameter", Communications in Statistics - Simulation and Computation, Vol. 32 No. 1, pp. 1-16.

10. Garg, A., Kalagnanam, J. R. (1998), "Approximations for the renewal function”, IEEE Transactions on Reliability, Vol. 47 No. 1, pp. 66-72.

11. Gertsbakh, I. (2000). Reliability Theory, With Applications to Preventive Maintenance, Berlin: Springer Verglag.

12. HU, X. (2006), "Approximation of partial distribution in renewal function calculation”, Computational Statistics \& Data Analysis, Vol. 50 No. 6, pp. 1615-1624.

13. Jardine, A. K. S. (1973). Maintenance, Replacement, and Reliability, London, Pitman.

14. Jardine, A. K. S., Tsang, A. H. C. (2006). Maintenance, Replacement, and Reliability: Theory and Applications, Boca Raton, CRC/Taylor \& Francis.

15. Jiang, R. (2008), "A Gamma-normal series truncation approximation for computing the Weibull renewal function", Reliability Engineering \& System Safety, Vol. 93 No. 4, pp. 616626.

16. Jiang, R. (2010), „A simple approximation for the renewal function with an increasing failure rate", Reliability Engineering \& System Safety, Vol. 95 No. 9, pp. 963-969.

17. Johnson, N. L, Kotz, S., Balakrishnan, N. (1994), Continuous Univariate Distributions, Volumes I and II, 2nd. Ed., New York: John Wiley and Sons.

18. Kottegoda, N. T., Rosso, R. (1997). Statistics, Probability, and Reliability for Civil and Environmental Engineers, New York: McGraw-Hill.

19. Lam, C. L. J., Le-Ngoc, T. (2006), „Estimation of typical sum of lognormal random variables using log shifted gamma approximation", IEEE Communications Letters, Vol. 10 No. 4, pp. 234- 235.

20. Nakagawa, T. (2011). Stochastic Processes: with Applications to Reliability Theory, London: Springer-Verlag.

21. O'Connor, A. N. (2011). Probability Distributions Used in Reliability Engineering, Maryland: RIAC.

22. Politis, K., Koutras, M. V. (2006), "Some new bounds for the renewal function”, Probability in the Engineering and Informational Sciences, Vol. 20 No. 2, pp. $231-250$.

23. Rinne, H. (2009). The Weibull Distribution: A Handbook, New York: CRC Press, Taylor \& Francis Group.

24. Robinson, N. I. (1997), „Renewal functions as series”, Stochastic Models, Vol. 13 No. 3, pp. $577-604$.

25. Romeo, M., Da Costa, V., Bardou, F. (2003), „Broad distribution effects in sums of lognormal random variables", The European Physical Journal B - Condensed Matter and Complex Systems, Vol. 32 No. 4, pp. 513-525.

26. Sheikh, A. K., Younas, M. (1985), "Renewal Models in Reliability Engineering", in Deopker, P. E. (Ed.), Failure and Prevention and Reliability, ASME, pp. 93-103.

27. Smeitink, E., Dekker, R. (1990), „A simple approximation to the renewal function”, IEEE Transactions on Reliability, Vol. 39 No. 1, pp. 71-75.

28. Tijms, H. C. (2003). A First Course in Stochastic Models, Chichester: John Wiley \& Sons.

29. van Noortwijk, J. M., van der Weide, J. A. M. (2008), „Applications to continuous-time processes of computational techniques for discrete-time renewal processes", Reliability Engineering \& System Safety, Vol. 93 No. 12, pp. 1853-1860.

\section{About the author}

Alenka Brezavšček (1967) is an assistant professor at the Faculty of Organizational Sciences, University of Maribor, Slovenia. Her research fields are stochastic processes, system reliability and availability, and information system security. Author can be reached at alenka.brezavscek@fov.uni-mb.si 